\title{
Decursin and Z-Ligustilide in Angelica tenuissima Root Extract Fermented by Aspergillus oryzae Display Anti-Pigment Activity in Melanoma Cells ${ }^{\mathbb{S}}$
}

\author{
Yuna Park ${ }^{1 \dagger}$, Dayoung Kim ${ }^{2 \dagger}$, Inho Yang ${ }^{3}$, Bomee Choi ${ }^{2}$, Jin Woo Lee ${ }^{1}$, Seung Namkoong ${ }^{1}$, Hyun Jung Koo ${ }^{4}$, \\ Sung Ryul Lee ${ }^{5}$, Myung Rye Park ${ }^{6}$, Hyosun Lim ${ }^{6}$, Youn Kyu Kim ${ }^{6 *}$, Sang-Jip Nam ${ }^{2 *}$, and Eun-Hwa Sohn ${ }^{1 *}$ \\ ${ }^{1}$ College of Health Science, Kangwon National University, Samcheok 25949, Republic of Korea \\ ${ }^{2}$ Department of Chemistry and Nano Science, Global Top 5 Program, Ewha Womans University, Seoul 03760, Republic of Korea \\ ${ }^{3}$ Department of Convergence Study on the Ocean Science and Technology, Korea Maritime and Ocean University, Busan 49112, Republic of Korea \\ ${ }^{4}$ Department of Medicinal and Industrial Crops, Korea National College of Agriculture and Fisheries, Jeonju 54874, Republic of Korea \\ ${ }^{5}$ Department of Convergence Biomedical Science, Cardiovascular and Metabolic Disease Center, College of Medicine, Inje University, Busan \\ 47392, Republic of Korea \\ ${ }^{6}$ Korea Research Institute Bio Science Co., Ltd., Yeoju 12668, Republic of Korea
}

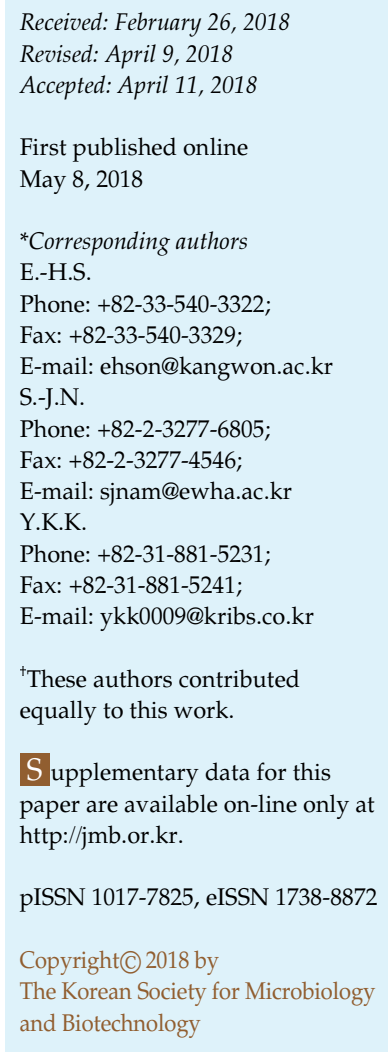

The anti-melanogenic effects of the extract of Angelica tenuissima (AT) root and the extract of AT root fermented by Aspergillus oryzae (FAT) were investigated. These effects were determined by measuring the inhibitory activity of AT and FAT on melanin production in B16F10 melanocytes and with in vitro tyrosinase activity assays. The AT extract inhibited melanin production at concentrations above $250 \mu \mathrm{g} / \mathrm{ml}$, and this inhibitory effect was significantly enhanced by the fermentation process with A. oryzae. HPLC analysis resulted in the isolation of two active compounds from both the AT and FAT extracts. Their chemical structures were identified as decursin and Z-ligustilide through comparison with previously reported NMR data. The decursin and Z-ligustilide contents were increased in the FAT extract and could be responsible for its enhanced inhibitory effects on melanin production and tyrosinase activity compared with that of the AT extract.

Keywords: Angelica tenuissima, decursin, Z-ligustilide, anti-melanogenic activity, Aspergillus oryzae, fermentation

\section{Introduction}

Pigmentation of the skin is one form of skin ageing and is associated with formation of reactive oxygen species, the effects of declining hormones, and ultraviolet radiation [1,
2]. Currently, cosmeceuticals are used to improve skin appearance, and many compounds, such as retinoids, and botanicals, like soy isoflavones, are used to develop safe cosmeceuticals [1]. Angelica tenuissima Nakai (Umbelliferae family) has been used in traditional Asian medicines to treat 
headaches and in remedies for women with gynecological diseases and anemia [3]. Recently, the interferon-mediated antiviral [4], anti-osteoporosis [5], and anti-inflammatory activities [6] of A tenuissima Nakai have been identified. However, the cosmetic uses of the crude extract of the A. tenuissima (AT) root and its fermented product (FAT) as a skin-whitening agent have not been investigated.

Skin depigmentation can be achieved by interfering with melanin synthesis either before, during, or after its production. Depigmentation is important for esthetic as well as medicinal purposes such as combating ageing of skin. Melanin is produced in melanosomes, which are a special organelle within melanocytes. The number of melanocytes is the same in all races, but the number and distribution of melanosomes in melanocytes determine race-related skin color [7]. Although melanin is a major factor in the determination of skin color and provides protection against ultraviolet-induced skin damage, an abnormal melanin content causes skin diseases or problems such as albinism, melasma, age spots, moles, and lentigo. In addition to producing an even and light skin tone, skinwhitening agents are also used in anti-ageing products, making them one of the most important and growing markets in cosmetics.

In a search for new skin-whitening ingredients, we investigated the crude extract of AT for its inhibitory effects against melanin production in mouse skin melanoma B16F10 cells after treatment with $\alpha$-melanin stimulating hormone $(\alpha-\mathrm{MSH})$. It has been widely recognized that fermentation of natural plants is considered as a good choice for reducing allergy-causing materials, as well as to enrich the bioavailability of beneficial anti-melanogenic agents $[8,9]$. Here, we used Aspergillus oryzae, which is a filamentous fungus used in traditional food processing and industrial applications, because of its known immunoreactivity-lowering potential [10]. The FAT created<smiles>CC(C)=CC(=O)O[C@H]1Cc2cc3ccc(=O)oc3cc2OC1(C)C</smiles>

1 decursin

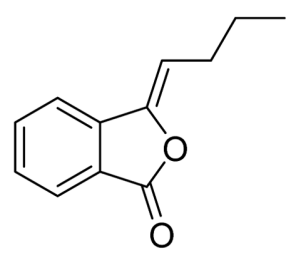

2 Z-ligustilide
Fig. 1. Chemical structures of decursin (1) and Z-ligustilide (2). by A. oryzae was also investigated for its anti-pigment activity. The enhanced anti-pigment activity of the FAT extract could result from its increased decursin and Z-ligustilide contents compared with the AT extract (Fig. 1). The mechanism of the anti-pigment effect of the FAT extract, decursin, and Z-ligustilide was elucidated to support the use of FAT as a new skin-whitening ingredient.

\section{Materials and Methods}

\section{Chemical and Reagents}

3-(4,5-Dimethylthiazol-2-yl)-2,5-diphenyltetrazolium bromide (MTT), $\alpha-\mathrm{MSH}$, decursin, and Z-ligustilide were purchased from Sigma-Aldrich (Korea). Unless indicated otherwise, all other chemicals were also obtained from Sigma-Aldrich Korea. A. oryzae was provided by Korea Research Institute Bio Science Co., Ltd. The primary antibodies against tyrosinase and $\beta$-actin were obtained from Santa Cruz Biotechnology (USA).

\section{Preparation of Angelica tenuissima Root Extract}

Angelica tenuissima Nakai roots were purchased from Jaechun Oriental Medicine Market and identified by Dr. S.C. Kang of Kyung Hee University. Voucher specimens were deposited at the herbarium of the School of Medicine at Kyung Hee University. The dried AT roots were chopped into small pieces and extracted in $70 \%$ ethanol at $80^{\circ} \mathrm{C}$ for $4 \mathrm{~h}$. The extract was subsequently filtered to remove any particulates and was concentrated under vacuum at $50^{\circ} \mathrm{C}$ to 53 Brix. The extract was lyophilized to obtain a powder and stored at $-20^{\circ} \mathrm{C}$ for further experiments. To prepare the FAT, fermentation was carried out in two steps: seed and main cultures. The seed cultures were prepared by inoculating $100 \mathrm{ml}$ of Luria-Bertani (LB) medium with A. oryzae in a 250-ml Erlenmeyer flask and incubating at $37^{\circ} \mathrm{C}$ for $48 \mathrm{~h}$. The main culture was prepared by adding $10 \%, 20 \%$, or $50 \%$ (weight/volume) of the AT root extract $(100 \mathrm{~g} / \mathrm{l})$ to $2 \mathrm{~L}$ of $\mathrm{LB}$ medium with $100 \mathrm{ml}$ of seed culture. Each batch was cultured at $37^{\circ} \mathrm{C}$ for $48 \mathrm{~h}$ with shaking at $50 \mathrm{rpm}$. The broth was extracted with ethanol (1:1 volume ratio) at $80^{\circ} \mathrm{C}$ for $4 \mathrm{~h}$, centrifuged at $2,000 \times g$ for $20 \mathrm{~min}$ at $4^{\circ} \mathrm{C}$, and filtered. The supernatant was concentrated and lyophilized to yield a dry extract of FAT. The AT root extract was prepared using the procedures described above without the addition of $A$. oryzae to the culture medium.

\section{Cell Culture}

B16F10 mouse skin melanoma cells were obtained from the American Type Culture Collection (ATCC, USA). The cells were cultured in Dulbecco's modified Eagle's medium (Gibco, USA) supplemented with $4 \mathrm{mM} \mathrm{L-glutamine,} 100 \mathrm{IU} / \mathrm{ml}$ penicillin, $100 \mu \mathrm{g} / \mathrm{ml}$ streptomycin, and $10 \%$ heat-inactivated fetal bovine serum (Gibco, USA). The cells were grown at $37^{\circ} \mathrm{C}$ in fully humidified air with $5 \% \mathrm{CO}_{2}$. 


\section{High-Performance Liquid Chromatography}

HPLC analysis was performed using an Agilent Technology 1100 HPLC system with a PDA detector and a reversed-phase analytical column (Phenomenex Luna C18(2), $5 \mu \mathrm{m}, 4.6 \times 100 \mathrm{~mm}$ ) at a flow rate of $1.0 \mathrm{ml} / \mathrm{min}$. HPLC-grade acetonitrile was purchased from Samchun Chemical (Korea) and used without further purification. Each lyophilized sample was fully dissolved in methanol with sonication for $30 \mathrm{~min}$. The injection volume for each sample was $20 \mu \mathrm{l}$ at a concentration of $500 \mathrm{mg} / \mathrm{ml}$, and $40 \%$ acetonitrile in deionized distilled water (with $0.1 \%$ trifluoroacetic acid as an acid buffer) was used as the isocratic solvent. The chromatograph was monitored at $310 \mathrm{~nm}$, and elution peaks for decursin and Z-ligustilide were observed at 26.8 and $21.3 \mathrm{~min}$, respectively. Each of the analyses was repeated three times.

\section{Melanin Production Inhibition Assay}

B16F10 cells were treated with $\alpha$-MSH $(100 \mathrm{nM})$ in the presence or absence of varying amounts of the FAT extract $(125-1,000 \mu \mathrm{M})$, decursin $(1-20 \mu \mathrm{M})$, or Z-ligustilide $(0.1-10 \mu \mathrm{g} / \mathrm{ml})$ for $48 \mathrm{~h}$. Arbutin $(100 \mu \mathrm{g} / \mathrm{ml})$ was used as a positive control. The culture medium was then removed, and the cells were washed with PBS and collected using centrifugation at 5,000 $\times g$ for $2 \mathrm{~min}$. The cell pellet was incubated with $200 \mu \mathrm{l}$ of $1 \mathrm{~N} \mathrm{NaOH}$ for $1 \mathrm{~h}$ at $60^{\circ} \mathrm{C}$, and the absorbance was measured at $405 \mathrm{~nm}$ using a microplate reader (Bio-Tek, USA).

\section{Tyrosinase Activity Assay}

Cellular tyrosinase activity was measured as previously reported [11]. Briefly, B16F10 cells were treated with $\alpha$-MSH $(100 \mathrm{nM})$ in the presence or absence of varying amounts of FAT extract (125$1,000 \mu \mathrm{M})$, decursin $(1-20 \mu \mathrm{M})$, or Z-ligustilide $(0.1-10 \mu \mathrm{g} / \mathrm{ml})$ for $48 \mathrm{~h}$. The culture medium was then removed, and the cells were washed with PBS and lysed with lysis buffer containing $50 \mathrm{mM}$ sodium phosphate ( $\mathrm{pH} 6.8$ ), $1 \%$ Triton $\mathrm{X}-100$, and $0.1 \mathrm{mM}$ PMSF. The membrane-bound tyrosinase was released from the melanosomes using detergent. The lysate was then centrifuged at $11,000 \times g$ for 15 min to obtain the supernatant containing the crude cellular tyrosinase. Forty micrograms of cell lysate was combined with $40 \mu \mathrm{l}$ of sodium phosphate buffer $(100 \mathrm{mM}, \mathrm{pH}$ 6.8) and $160 \mu \mathrm{l}$ of L-DOPA (10 mM) for $1 \mathrm{~h}$. The absorbance was then measured at $490 \mathrm{~nm}$ using a microplate reader.

\section{Immunoblot Analysis}

B16F10 cell extracts were prepared and western blotting was performed. The protein content of the whole-cell lysates was measured using a BCA protein assay (Bio-Rad Laboratories, USA) with bovine serum albumin as a standard. The samples were diluted with $1 \times$ radio-immunoprecipitation lysis buffer containing $1 \% \beta$-mercaptoethanol. Equal amounts of cellular protein $(50 \mu \mathrm{g})$ were resolved using sodium dodecyl sulfate-polyacrylamide gel electrophoresis (SDS-PAGE) on $10 \%$ polyacrylamide gels and transferred to nitrocellulose membranes. After blocking with a protein blocking buffer, the membranes were incubated with the primary antibody and then with the horseradish peroxidaseconjugated IgG secondary antibody. The immunoreactive proteins were visualized using the ECL western blot detection system (Amersham Pharmacia Biotech, USA). The protein levels were determined relative to the $\beta$-actin loading control.

\section{Statistical Analysis}

Each experiment was repeated either three or four times. For qualitative data, the results of a representative experiment are shown. For quantitative data, the results are expressed as the mean \pm SEM and were analyzed by one-way ANOVA followed by Turkey's method using SigmaPlot software (Systat Software Inc., USA). A statistical probability of $p<0.05$ was considered significant.

\section{Results}

\section{Effects of AT and FAT Extracts on Melanin Production in B16F10 Cells}

The inhibitory effects of AT and FAT were investigated because the suppression of melanin production in the skin is a primary target of depigmenting agents $[12,13]$. Because arbutin behaves as a competitive inhibitor of tyrosinase through L-tyrosine or L-DOPA [14], it was used as a positive control for inhibition of melanin production. As shown in Fig. 2A, treatment of $\alpha$-MSH-stimulated B16F10 cells with AT extract above $250 \mu \mathrm{g} / \mathrm{ml}$ resulted in significant suppression of melanin production $(p<0.05)$. This result implies that the AT extract possesses inhibitory potential against melanin production. We also prepared fermented products of AT using A. oryzae to augment the inhibitory activity of AT against melanin production. FAT10, FAT20, and FAT50 represent extracts made from $10 \%, 20 \%$, or $50 \%$ (w/v) AT, respectively, fermented with A. oryzae (Table S1). AT20, which was prepared from $20 \%(\mathrm{w} / \mathrm{v})$ AT extract in LB medium, was used as the control (Table S1). As shown in Figs. $3 \mathrm{~A}$ and $3 \mathrm{~B}$, inhibition of melanin production was observed with FAT10, FAT20, and FAT50. As indicated in the figure, FAT20 was better at inhibiting melanin production than AT20. Half maximal inhibitory concentration $\left(\mathrm{IC}_{50}\right)$ values of the AT20 and FAT20 extracts on $\alpha$-MSH-induced melanin production were also determined (Fig. 3B), and the $\mathrm{IC}_{50}$ value of FAT20 was half that of AT20.

\section{Effects of FAT on the Expression and Activity of Tyrosinase}

Tyrosinase has been extensively used as a target enzyme to search for depigmenting agents, since this enzyme is a rate-limiting enzyme in melanogenesis [12]. When the protein expression level of tyrosinase was determined using immunoblots after stimulation with $\alpha-\mathrm{MSH}$, there 


\section{A}

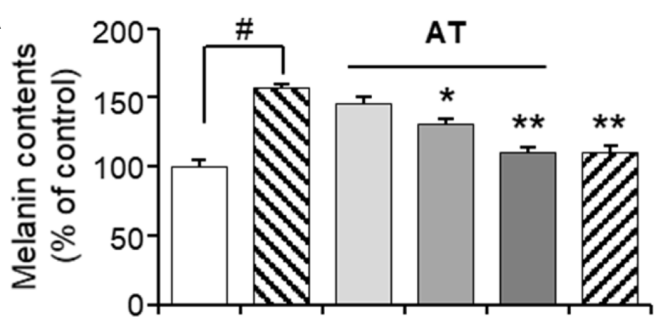

B

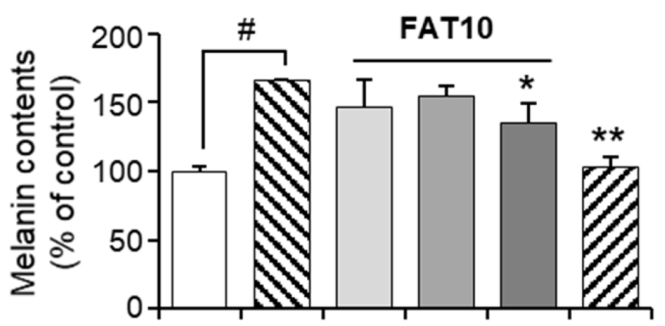

C

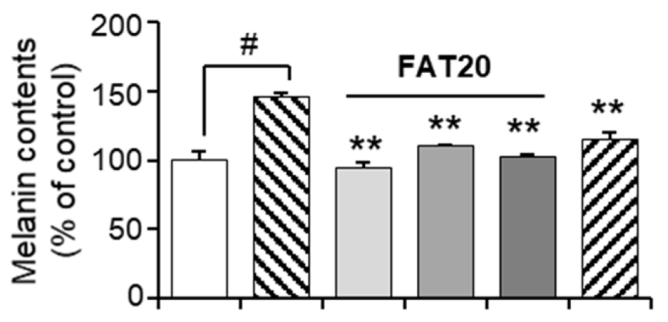

D

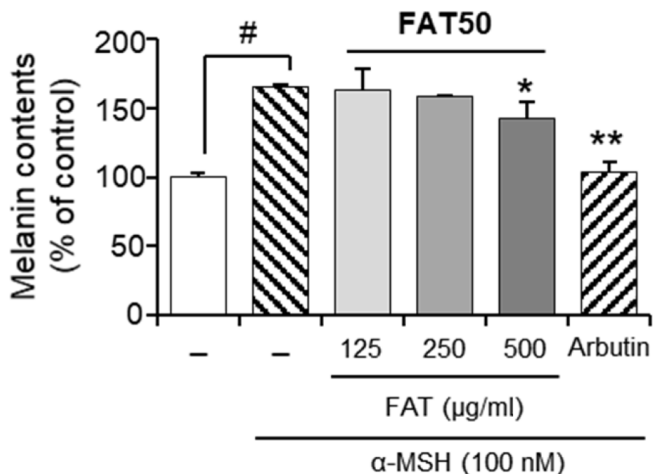

Fig. 2. Melanin production in B16F10 cells cultured in the presence or absence of extracts of Angelica tenuissima (AT) root and fermented AT (FAT) for $48 \mathrm{~h}$ after $\alpha$-melanocyte stimulating hormone $(\alpha-\mathrm{MSH})$ insult.

Relative melanin content after treatment with (A) AT, (B) FAT10, (C) FAT20, and (D) FAT50. ${ }^{*} p<0.05,{ }^{* *} p<0.01$ versus $\alpha$-MSH-treated control and $\# p<0.05$ versus untreated control.

were no significant changes in the protein expression level of tyrosinase in the presence of up to $250 \mu \mathrm{g} / \mathrm{ml}$ of FAT20. A significant reduction in tyrosinase expression was shown only at $500 \mu \mathrm{g} / \mathrm{ml}$ of FAT20 (Fig. 3C). Because tyrosinase activity also plays a role in melanogenesis, we investigated the effects of FAT on tyrosinase enzyme activity using a photometric method. In accordance with the results from
Fig. 3C, treatment with FAT20 resulted in a dose-dependent reduction in the cellular tyrosinase activity (Fig. 3D). These results indicated that the inhibitory effects of FAT20 on the production of melanin are not associated with suppression of tyrosinase protein expression (Fig. 3C) but with inhibition of its enzymatic activity (Fig. 3D).

\section{Increase in Decursin and Z-Ligustilide Contents by} Fermentation with A. oryzae

The AT and FAT extracts displayed inhibitory activity toward melanin production (Figs. 2 and 3). Therefore, the major components responsible for this bioactivity were further identified by reversed-phase HPLC analysis. We identified two active compounds, decursin and Z-ligustilide, through comparisons with NMR data from the literature and the HPLC retention times of commercial standards (calibration curves for standards, see Figs. S1 and S2). Fermentation of the AT extract with A. oryzae increased the amounts of decursin (140-367\%) and Z-ligustilide (103$281 \%$ ) (Table 1).

Effects of Decursin and Z-Ligustilide on the Production of Melanin and Tyrosinase Activity in B16F10 Cells

To confirm the melanin production-inhibition activity of decursin and Z-ligustilide, the melanin production rate and in vitro tyrosinase activity were determined in the presence of decursin or Z-ligustilide (Fig. 4). As shown in Table 1, the 20\% FAT (FAT20) contains more content of Z-ligustilide than that of decursin and 20\% AT (AT20) (Table 1). We speculated that the inhibitory effect of FAT20 on melanin production (Fig. 3) might be influenced by the action of $Z$-ligustilide. Thus, we tested the effect of $Z$-ligustilide with a higher concentration $(0.5-50 \mu \mathrm{M})$, compared with decursin $(1-20 \mu \mathrm{M})$, on melanin production and tyrosinase activity.

Decursin suppressed the production of melanin significantly, even at the lowest concentration tested $(1 \mu \mathrm{M}$, $p<0.05)$, but the tyrosinase-inhibition activity of decursin was only observed at a concentration of $20 \mu \mathrm{M}$ (Figs. 4A and $4 \mathrm{C}$ ). These results suggest that decursin might be a potent inhibitor of melanin production, but not through tyrosinase inhibition. However, Z-ligustilide showed tyrosinase-inhibition activity at $50 \mu \mathrm{M}$ (Fig. 4D). Therefore, we suggest that decursin and Z-ligustilide are the active compounds involved in the inhibition of melanin production and tyrosinase activity, respectively.

\section{Discussion}

As one of the indicators of youth and beauty, clear skin is 
A

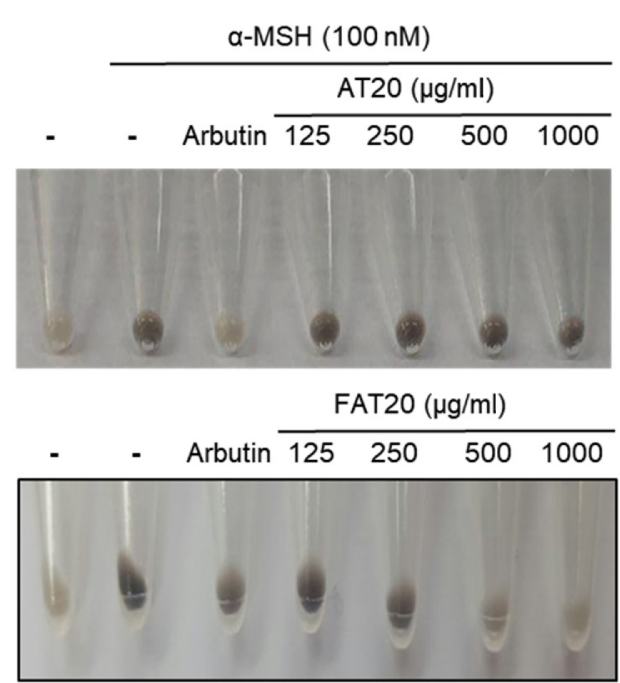

B

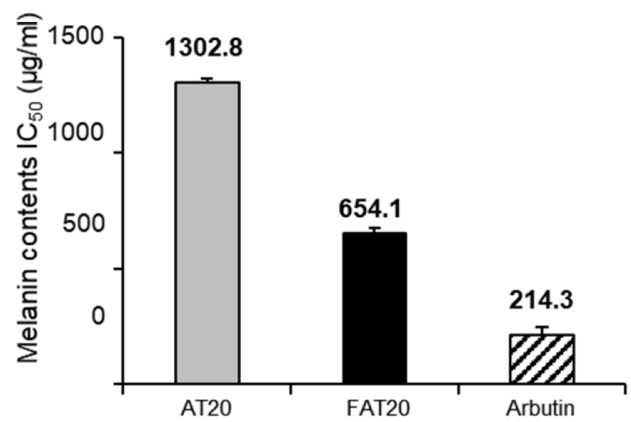

C

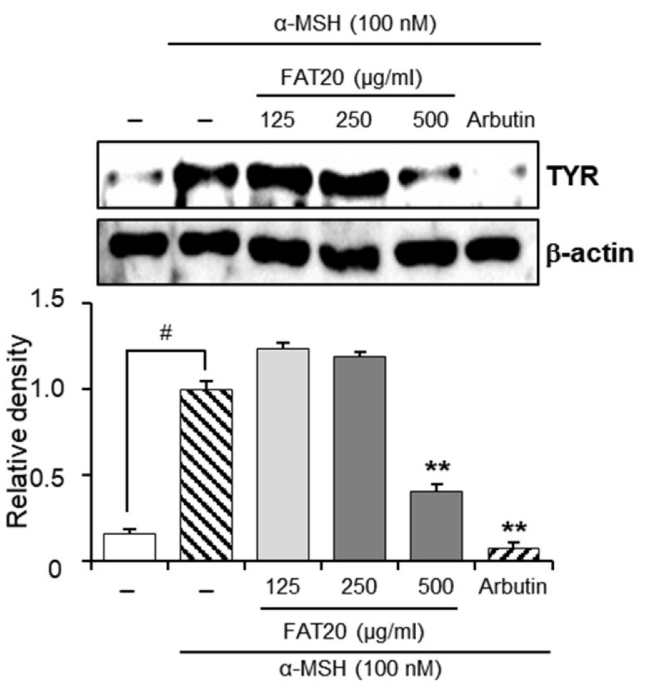

D

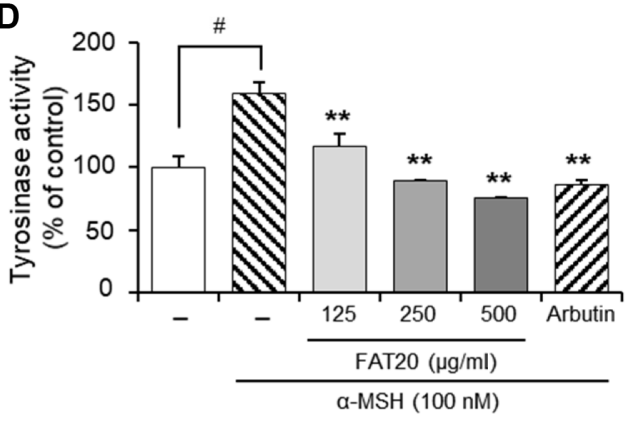

Fig. 3. Inhibitory effect of fermented $20 \%$ Angelica tenuissima (AT) root extract (FAT20) on melanin production and tyrosinase activity.

(A) Images of pellets of harvested B16F10 cells and determination of the relative melanin contents after treatment with the extract of AT20 and FAT2 0 for $48 \mathrm{~h}$. (B) The $\mathrm{IC}_{50}$ values of the extracts of AT20 and FAT20 on $\alpha$-MSH-induced melanin production. (C, D) Effects of the extract of FAT20 on (C) tyrosinase protein expression and (D) tyrosinase activity in B16F10. Cells were treated with the indicated concentrations of AT 20 and FAT20 for $48 \mathrm{~h}$, and then total cell lysates were subjected to western blot or colorimetric method. Bar graphs show the densitometric evaluation. Data are the mean \pm SEM and expressed as a percentage of the untreated control. ${ }^{* *} p<0.01$ versus $\alpha$-MSH-treated control and $\# p<0.05$ versus untreated control.

a very important part of a satisfactory appearance, especially for Asian women [15]. Melanin is a primary factor in skin color [13], and increased production of cutaneous melanin may cause esthetic problems such as hyperpigmentary conditions [15]. In addition to treating skin pigmentation problems, the interest in skin-whitening ingredients

Table 1. Yield of decursin and Z-ligustilide of AT and FAT.

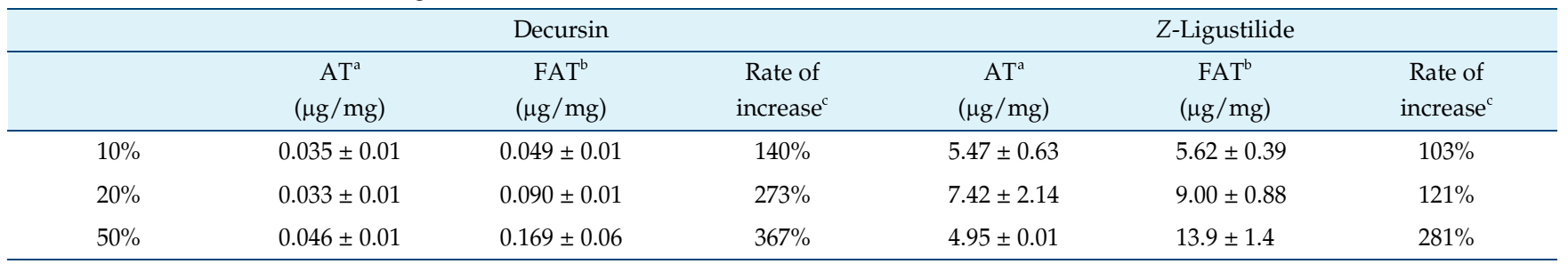

${ }^{\mathrm{a}}$ AT: extract of Angelica tenuissima. ${ }^{\mathrm{b}} \mathrm{FAT}$ : fermented extract of A. tenuissima. ${ }^{\mathrm{c}}$ Rate of increase was determined as (yield of FAT)/(yield of AT) at same initial AT extract. 
A
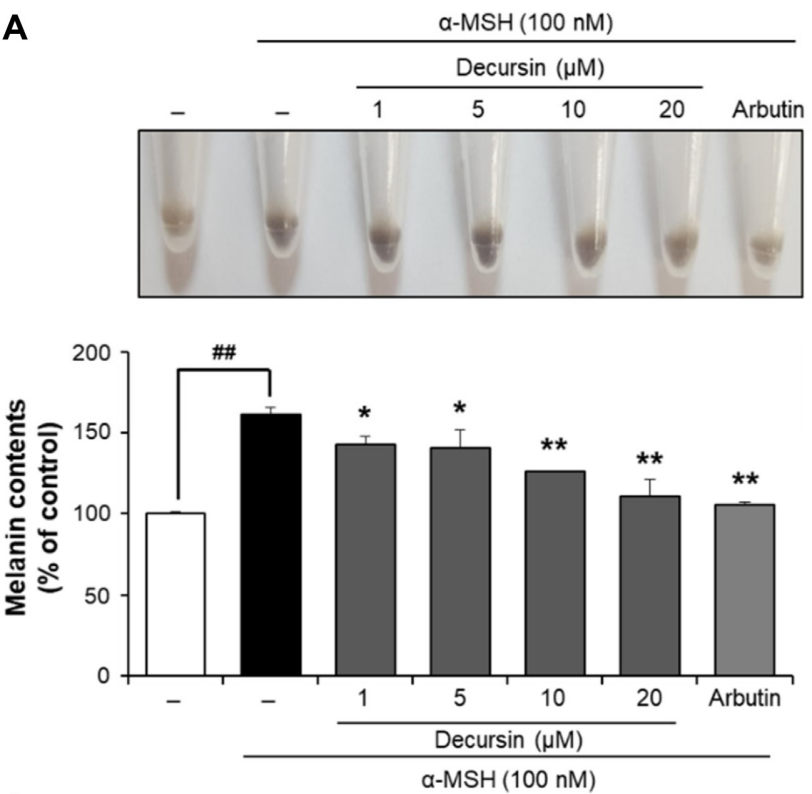

C

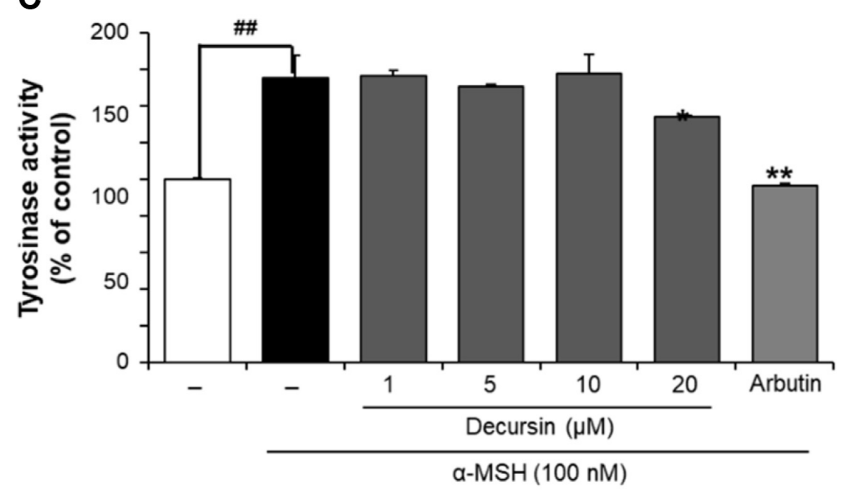

B
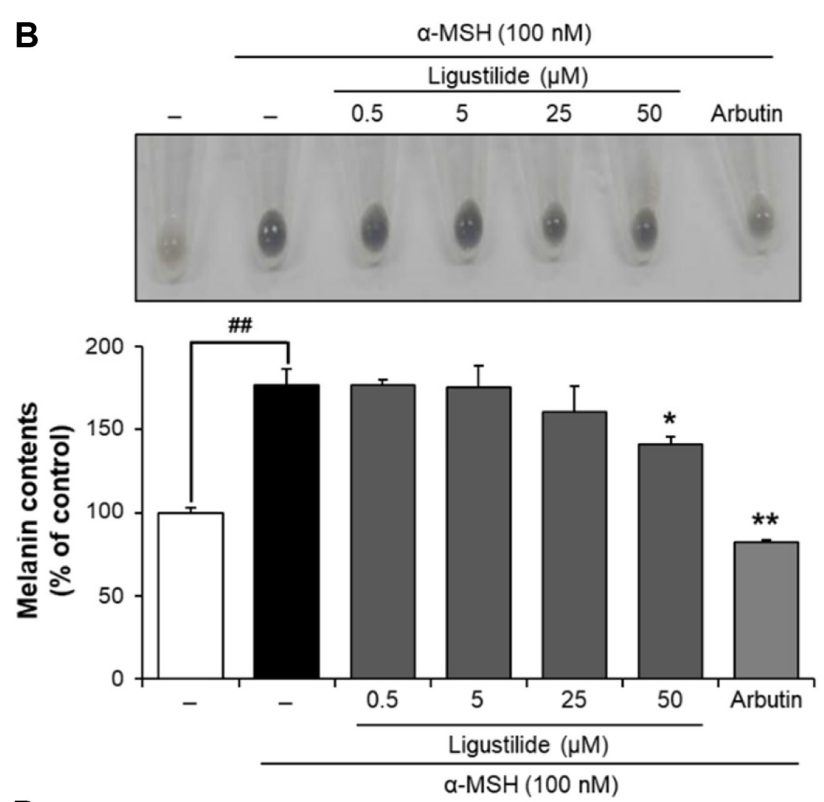

D

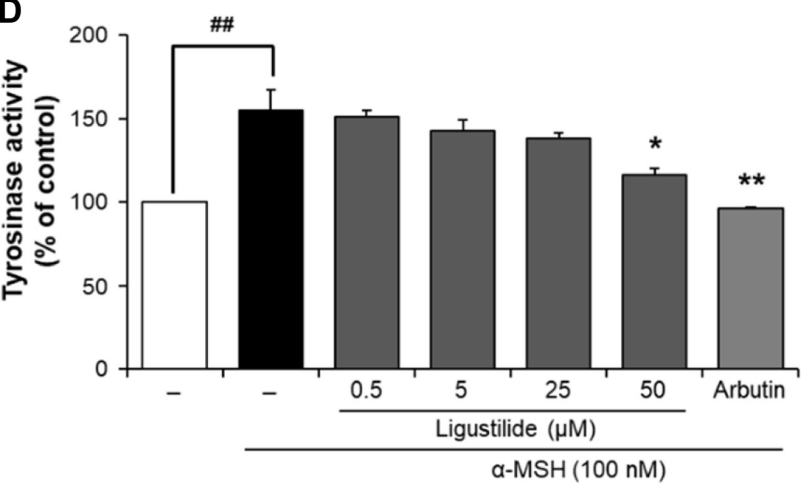

Fig. 4. Effects of decursin and Z-ligustilide on melanogenesis in B16F10 cells.

B16F10 cells were cultured in the presence or absence of decursin and Z-ligustilide for $48 \mathrm{~h}$ after $\alpha$-MSH insult. (A, B) Images of pellets of harvested B16F10 cells and determination of the relative melanin contents after treatment of (A) decursin and (B) Z-ligustilide. Inhibitory effects of (C) decursin and (D) Z-ligustilide on tyrosinase activity. The tyrosinase activity was determined by a colorimetric method. Data are the mean \pm SEM and expressed as a percentage of untreated control. ${ }^{*} p<0.05,{ }^{* *} p<0.01$ versus $\alpha$-MSH-treated control and \#\#p $<0.01$ versus untreated control.

with antioxidant and anti-ageing properties has grown tremendously. It has been suggested that melanin production in melanocytes and the skin can be reduced by interrupting melanin synthesis, which is mediated by tyrosinase transport and removal through skin turnover $[15,16]$. Hydroquinone, a tyrosinase inhibitor, is one of the most potent skin-whitening agents, but some adverse effects of long-term treatment have been identified [17]. Although arbutin and kojic acid are currently effective in controlling pigmentation, there is a growing interest in finding alternative agents with lower mutagenic potential [17] from natural products because medicinal plants have historically proven value as a source of molecules with therapeutic potential [18].

In this study, the extract of Angelica tenuissima root showed an inhibitory effect against melanin production at $250 \mu \mathrm{g} / \mathrm{ml}$. This inhibitory effect was significantly enhanced after the AT root extract was fermented by A. oryzae. The extract of the fermented product, FAT20, significantly reduced the melanin content and suppressed tyrosinase activity.

Based on HPLC analysis, we identified decursin and $Z$-ligustilide as the active compounds, and the contents of these two compounds were increased in FAT20 compared with AT20. Decursin significantly decreased melanin production at a concentration of $1 \mu \mathrm{M}$, but its tyrosinase- 
inhibition activity was only observed at a concentration of $20 \mu \mathrm{M}$. These results suggest that decursin has an inhibitory effect on melanin production in B16F10 cells in a tyrosinase activity-independent manner (Fig. 4C). In a previous study, decursin was found to exhibit anticancer activity in B16F10 melanoma cells [19]. However, it was not cytotoxic to B16F10 cells at concentrations up to $20 \mu \mathrm{M}$ in this study. This implies that the anti-melanogenic activity of decursin is not related to its cytotoxicity toward B16F10 cells. Additionally, Z-ligustilide displayed high inhibitory activity against tyrosinase, but with a moderate reduction in melanin content compared with decursin (Fig. 4).

Although the tyrosinase-inhibition activities of decursin and Z-ligustilide are not solely responsible for the antipigmentation activity of the FAT extract, we identified them as the primary anti-pigment compounds of the FAT extract in this study. It is possible that unidentified active compounds that are enriched in the fermentation process could also display anti-pigmentation effects. Furthermore, identification of the underlying mechanisms involved in the anti-melanogenic effects of FAT20, decursin, and Z-ligustilide, such as melanocyte maturation and effects on distribution through inhibition of melanosome transfer or melanin dispersion, remains for further study.

\section{Acknowledgments}

This research was supported by the Ministry of Trade, Industry \& Energy (MOTIE), Korea Institute for Advancement of Technology (KIAT) through the Encouragement Program for The Industries of Economic Cooperation Region.

\section{Conflict of Interest}

The authors have no financial conflicts of interest to declare.

\section{References}

1. Pham AK, Dinulos JG. 2014. Cosmeceuticals for children: should you care? Curr. Opin. Pediatr. 26: 446-451.

2. Desmedt B, Courselle P, De Beer JO, Rogiers V, Grosber M, Deconinck E, et al. 2016. Overview of skin whitening agents with an insight into the illegal cosmetic market in Europe. J. Eur. Acad. Dermatol. Venereol. 30: 943-950.

3. Han BH, Sung CK, Takeatsu K, But PPH, Guo J-X. 1998. International Collation of Traditional and Folk Medicine. World Scientific Publishing Co. Pte. Ltd., Singapore.

4. Weeratunga $\mathrm{P}$, Uddin MB, Kim MS, Lee BH, Kim TH, Yoon JE, et al. 2016. Interferon-mediated antiviral activities of Angelica tenuissima Nakai and its active components. J.
Microbiol. 54: 57-70.

5. Ahn SJ, Baek JM, Cheon YH, Park SH, Lee MS, Oh J, et al. 2015. The inhibitory effect of Angelica tenuissima water extract on receptor activator of nuclear factor-kappa-B ligand-induced osteoclast differentiation and bone resorbing activity of mature osteoclasts. Am. J. Chin. Med. 43: 715-729.

6. Lee SH, Choi H, Kim H, Lee H, Sung YH, Kim SE, et al. 2010. Inhibitory effect of Angelicae Tenuissimae Radix on expressions of cyclooxygenase-2 and inducible nitric oxide synthase in mouse BV2 microglial cells. Neurol. Res. 32 Suppl 1: 58-63.

7. Desmedt B, Van Hoeck E, Rogiers V, Courselle P, De Beer JO, De Paepe K, et al. 2014. Characterization of suspected illegal skin whitening cosmetics. J. Pharm. Biomed. Anal. 90: 85-91.

8. Shin YK, Son HU, Kim JM, Heo JC, Lee SH, Kim JG. 2015. Cinnamomum cassia bark produced by solid-state fermentation with Phellinus baumii has the potential to alleviate atopic dermatitis-related symptoms. Int. J. Mol. Med. 35: 187-194.

9. Kim OK, Chang JY, Nam DE, Park YK, Jun W, Lee J. 2015. Effect of Canavalia gladiata extract fermented with Aspergillus oryzae on the development of atopic dermatitis in $\mathrm{NC} / \mathrm{Nga}$ mice. Int. Arch. Allergy Immunol. 168: 79-89.

10. Frias J, Song YS, Martinez-Villaluenga C, Gonzalez de Mejia E, Vidal-Valverde C. 2008. Immunoreactivity and amino acid content of fermented soybean products. J. Agric. Food Chem. 56: 99-105.

11. Oh SW, Park S-H, Lee HS, Kang M, Lee SE, Yoo JA, et al. 2017. Melanogenic mechanism of ethanolic extract of Dalbergia odorifera. Mol. Cell. Toxicol. 13: 453-459.

12. Gillbro JM, Olsson MJ. 2011. The melanogenesis and mechanisms of skin-lightening agents - existing and new approaches. Int. J. Cosmet. Sci. 33: 210-221.

13. Videira IF, Moura DF, Magina S. 2013. Mechanisms regulating melanogenesis. An. Bras. Dermatol. 88: 76-83.

14. Maeda K, Fukuda M. 1996. Arbutin: mechanism of its depigmenting action in human melanocyte culture. J. Pharmacol. Exp. Ther. 276: 765-769.

15. Smit N, Vicanova J, Pavel S. 2009. The hunt for natural skin whitening agents. Int. J. Mol. Sci. 10: 5326-5349.

16. Briganti S, Camera E, Picardo M. 2003. Chemical and instrumental approaches to treat hyperpigmentation. Pigment Cell Res. 16: 101-110.

17. DeCaprio AP. 1999. The toxicology of hydroquinone relevance to occupational and environmental exposure. Crit. Rev. Toxicol. 29: 283-330.

18. Atanasov AG, Waltenberger B, Pferschy-Wenzig EM, Linder T, Wawrosch C, Uhrin P, et al. 2015. Discovery and resupply of pharmacologically active plant-derived natural products: a review. Biotechnol. Adv. 33: 1582-1614.

19. Kim BS, Seo H, Kim HJ, Bae SM, Son HN, Lee YJ, et al. 2015. Decursin from Angelica gigas Nakai inhibits B16F10 melanoma growth through induction of apoptosis. J. Med. Food 18: 1121-1127. 